\title{
Combinatorial Auction Based Channel Allocation in Cognitive Radio Networks
}

\author{
Wei Zhou ${ }^{1}$, Tao Jing ${ }^{1}$, Wei Cheng ${ }^{2}$, Tao Chen ${ }^{3}$, Yan Huo ${ }^{1}$ \\ ${ }^{1}$ School of Electronics and Information Engineering, Beijing Jiaotong University, Beijing, China \\ ${ }^{2}$ Department of Computer Science, University of Massachusetts Lowell, Lowell MA, USA \\ ${ }^{3}$ VTT Technical Research Centre of Finland, Oulu, Finland \\ E-mail: ${ }^{1}\left\{11111032\right.$, tjing, yhuo\}@bjtu.edu.cn, ${ }^{2}$ wcheng@cs.uml.edu, ${ }^{3}$ tao.chen@vtt.fi
}

\begin{abstract}
Combinatorial auction with flexible bidding formats is proposed in this paper for the channel allocation problem in cognitive radio networks. Different from other auction mechanisms, our solution includes the channel characteristic and user requirements in the biding, and therefore has more freedom to reflect the preference of secondary users on the channel selection. Four bidding formats are introduced, each of which has different emphasis to maximize social welfare of the spectrum resource. The details of each bidding format are explained, followed by the optimal solution or approximation solution proposed for each format. The approximation ratio of proposed algorithms is analyzed. Finally a numerical test is conducted in terms of social welfare and spectrum utilization. The test shows that actual approximation ratio is close to the optimal one.
\end{abstract}

\section{INTRODUCTION}

Among methods for the allocation of channel access rights in cognitive radio networks (CRN), auction [1] has been widely used, along with others as price [2], game theory [3], and etc. Although fairness and efficiency are shown, [4] [5] [6] [7], most of auction approaches do not consider well the SU's preference on channels. For instance, in [4], the authors formulates the auction framework as a non-cooperative game, but assumes each SU can only connect to a single PU; in [5], each user is only allowed to access one spectrum band; in [6], a multi-user multi-unit double auction mechanism is proposed, but channels are treated homogeneously across SUs.

The combinatorial auction appears to be attractive for the channel allocation in CRN, as it considers the trade of multiple items in bundles. However, there are few literatures on the combinatorial auction in CRN. Even fewer researches studied the flexibility of SU's requirements on the channel selection. In [7], SUs are allowed to bid a bundle of timefrequency blocks. However it has limitation to represent SU's specific requirements. For instance, the channel $c_{1} c_{2} c_{3}$ are the same in bandwidth and characteristic but only one of them is preferred. To solve this problem, we define four bidding formats. The basic format is named atomic bid. With the help of two operators $A N D$ and $O R$, we define other three formats as alternative bid, additive bid and integrated bid. With those formats, we are able to define a bidding structural uniformity which adequately describes SU's requirements on the channel selection.

In the paper, we model the channel as an item in the combinatorial auction. We consider the channel characteristic as a parameter in the auction. In addition, SU's valuations on channel are channel-dependent. SU determines its bid based on transmission demand and valuations. We strict an SU's bid to four defined formats with allowing each bidder request multiple bundles in the bid and put an arbitrary combination of items in a bundle. Our paper targets following problem: determine the winners in channel allocation to achieve a high social welfare (the sum of winners' valuations). We first illustrate the optimal solution under the atomic and alternative bid. Then we propose a greedy algorithm under the additive bid and briefly analyze the approximation ratio of the algorithm. Finally we propose an approximation algorithm under integrated bid based on the solutions in the alternative and additive bid.

The rest of the paper is organized as follows. The system model, preliminary knowledge on valuation and objective are introduced in Section II. Four bidding formats are described in Sections III. The channel allocation algorithm under different bidding formats are presented in Section IV. The numerical evaluations are given in Section V. Finally the conclusion is drawn in Section VI.

\section{PRELIMINARY}

\section{A. System Model}

As illustrated in Fig. 1, we consider $m$ primary users (PU) and $n$ SUs denoted as $\mathcal{S}=\left\{s_{1}, s_{2}, \ldots, s_{j}, \ldots, s_{n}\right\}$, randomly distributed in a given area. These SUs are connected to an information agent, which acts as an auctioneer for bidding. Each PU operates on a channel orthogonal with the channels of other PUs. Therefore $m$ channels is available in this area, denoted by $\mathcal{C}=\left\{c_{1}, c_{2}, \ldots, c_{i}, \ldots, c_{m}\right\}$. In our model, these channels differ not only on ownership, but also on channel characteristic.

SUs is allowed to purchase permission to access the channels owned by PUs. We assume that a channel is the basic unit that can be lent by an SU. Furthermore we adopt the underlay spectrum sharing model in which an SU can access the channel simultaneously even the PU is transmitting, as long as the interference to the PU is below a threshold. Each PU declares a tolerant interference level to the information agent before leasing out and the agent publishes these thresholds to all SUs. We further assume an SU can calculate its transmitting power on a channel owned by an active PU according to the interference threshold and distance to PU.

\section{B. Valuation}

1) Single Channel: Each $\mathrm{SU} j$ has a set of channeldependent valuations which describe the highest price $\mathrm{SU} j$ is willing to pay for each channel. The valuation $v_{j}^{i}$ of $\mathrm{SU} j$ 


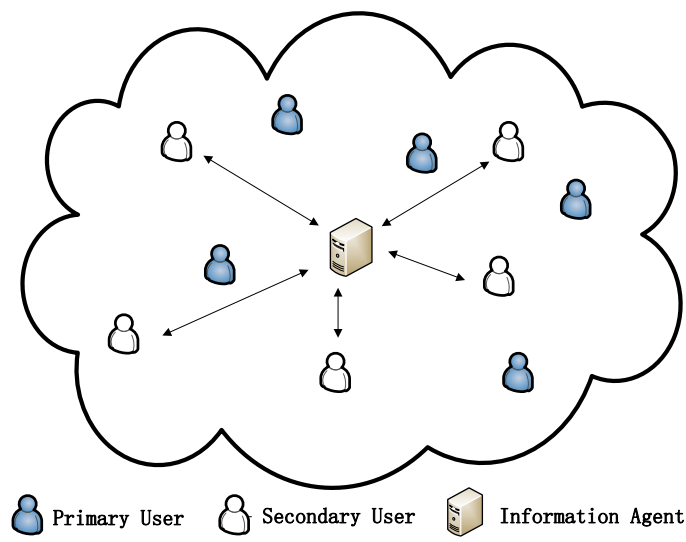

Figure 1: System Model

on channel $i$ is evaluated according to metrics like data rate and consecutive stable transmitting time. The valuation integrates all these metrics in a function, as

$$
v_{j}^{i}=\sum_{l} \alpha_{j}^{l} M_{j}^{i, l}
$$

$M_{j}^{i, l}$ is the value of metric $l$ of SU $j$ on channel $i . \alpha_{j}^{l}$ is the coefficient SU $j$ giving to metric $l$, which depends on SU's preference and transmission request. From 1, we can easily derive that, an SU's valuations differ on each channel and so are the valuations of different SUs on a single channel.

The value of a metric is determined by the attributes of the metric. Three main attributes affecting the valuation of a channel are listed below:

- The usable power level on the channel when PU is active.

- The channel characteristic, e.g., central frequency and bandwidth.

- The level of activity of PU on the channel. It is evident that longer the PU using the channel, the less stable slots an SU can get. Although a PU and an SU share a channel, the SU can transmit in a higher power when the PU is inactive.

A metric can be valued by its attributes, and then forms the valuation of a channel.

2) subset of channels: In practice, it is possible for an SU to put a request on a subset of channels, thus it should have a single valuation on the subset. In general, there are two kinds of subsets [8]: complement [9], where an SU may value the set of items higher than the sum of the individual values of the items, and substitute [10], where an SU may value the set of items lower than the sum of the individual values of the items. In our study, we allow both types of subsets in the auction.

\section{Problem Formulation}

Both PUs and SUs have incentives to trade: PU can get profit and SU can access the channel. In the trade, an SU forms its channel request by taking account of its transmission demand and channel-dependent valuations. We study how to allocate channels to SUs so as to maximize the social welfare.
We apply an auction framework to study this problem. SUs are buyers, who submit bids to the information agent. The buyer's bid consists of arbitrary bundles of channels and corresponding valuations. After receiving bids, the auctioneer determines the final allocation. Due to the differences on channel characteristic and on SUs' requirements, we formulate the allocation problem as a combinatorial auction problem [11]. The combination auction is defined as an auction in which bidders can place bids on combination of items, called "bundle" or "package", which satisfy two conditions: no good is assigned to more than one buyer, and no buyer receives more than one bundle of items [7]. In our study, we extend the concept of bundle, which can be either a single channel or a combination of channels.

We aim to design a combinatorial auction mechanism for channel allocation: for each buyer $j$ submits a bid $b_{j}$, containing a set of bundles $T_{j}^{e} \subseteq \mathcal{C}, e \in\left\{1,2, \ldots, E_{j}\right\} . E_{j}$ is the number of bundles in $b_{j}$. Let $v_{j}\left(T_{j}^{e}\right)$ be valuation of buyer $j$ for bundle $T_{j}^{e}$. Moreover we use a binary variable $X\left(j, T_{j}^{e}\right)$ to denote the allocation result, with 1 indicates buyer $j$ wins bundle $T_{j}^{e}$ and 0 indicates the buyer loses. Then the objective is to

$$
\begin{aligned}
\max & \sum_{j \in \mathcal{S}} \sum_{e=1}^{E_{j}} v_{j}\left(T_{j}^{e}\right) X\left(j, T_{j}^{e}\right), T_{j}^{e} \subseteq \mathcal{C} \\
\text { s.t. } & \sum_{e=1}^{E_{j}} X\left(j, T_{j}^{e}\right) \leqslant 1, \forall j \in \mathcal{S} \\
& T_{j}^{e} \cap T_{j^{\prime}}^{e^{\prime}}=\emptyset, \forall X\left(j, T_{j}^{e}\right)=1, X\left(j^{\prime}, T_{j^{\prime}}^{e^{\prime}}\right)=1 \\
& \bigcup_{j \in \mathcal{S}} \bigcup_{X\left(j, T_{j}^{e}\right)=1} T_{j}^{e} \subseteq \mathcal{C}
\end{aligned}
$$

The goal is to maximize the sum of winning buyers' valuations. The first constraint means a buyer can get at most one bundle of channels; the second constraint indicates that there is no intersection between any two winning bundles, i.e., every channel should only be allocated once; the third constraint means all the allocated bundles should not exceed the channel set. In following section, we use $S U$, buyer and bidder interchangeably.

\section{BIDDING FORMAT}

In this section we define four bidding formats, by which we could fully represent varieties on buyers' requirements. We use $b_{j}$ to denote the submitted bid of buyer $j$ in the description of each format.

\section{A. Single unit atomic bid}

Each buyer submits its bid $b_{j}$, including the request on single channel and specific valuation, as $\left(c_{i}, v_{j}^{i}\right)$. This is the basic unit of bidding, including a single bundle in the bid and only one channel in the bundle.

\section{B. Multi-unit alternative bid}

Each buyer submits a bid $b_{j}$, including a number of atomic bids, i.e., a collection of pairs $\left(c_{i}, v_{j}^{i}\right)$. In this case, the buyer requests multiple bundles with single channel in each bundle. These bundles are substitutable, which means the buyer is willing to accept one but only one bundle in its bid. We use 
the "OR" operator to express the alternation in request. For example, buyer $j$ bids

$$
\left(c_{1}, v_{j}^{1}\right) O R\left(c_{2}, v_{j}^{2}\right)
$$

to express bidding price $v_{j}^{1}$ on $c_{1}$ and $v_{j}^{2}$ on $c_{2}$, and the willingness to pay either $c_{1}$ or $c_{2}$. It is suitable for the case when a wireless device locates in an area with PUs sparsely distributed. The buyer has many available channels while a single channel is able to fulfill its spectrum requirement.

\section{Multi-unit additive bid}

Each buyer submits its bid $b_{j}$, including a request on a subset of channels and a single valuation. In this case, buyer submits a single bundle, but there are multiple channels in the bundle. We express this additive request by "AND" operator. For example, if a buyer $j$ has a request on channel $c_{1}$ and $c_{2}$ with the valuation $v_{j}^{12}$, it will submit an "AND" bid as

$$
\left(c_{1} A N D c_{2}, v_{j}^{12}\right) \text {. }
$$

This bid can be used by wireless devices with multiple radios, which can transmit and receive data simultaneously on different channels, or wireless devices using frequency hopping.

\section{Integrated bid}

Due to the complexity of the network environment, a user may like to express more complex bids, for example,

$$
c_{1} A N D\left(c_{2} O R c_{3}\right)
$$

The buyer requests for two channels: one is $c_{1}$, but for the other one, it has no preference between $c_{2}$ and $c_{3}$. Under this format, bids may not be directly composed by separate bundles, like (5). We cannot directly extract bundles from the bid, but we could change the bid into

$$
\left(c_{1} A N D c_{2}\right) O R\left(c_{1} A N D c_{3}\right) \text {. }
$$

In (6), it is clear that the buyer submits two bundles and both contain two channels. We extract the bundle information without misrepresenting the origin request of buyer.

As illustrated in (2), the final allocation is decided according to specific valuations of all bundles. If a bid is constructed as (5) or even more complex, we cannot obtain accurate valuations from the origin request, which may lead to a significant degradation on efficiency. Therefore we strict the bidders to reconstruct their bids into a uniform structure before submitting to the auctioneer: No matter which form the request is, it can be reconstructed to OR combination of atomic bids and additive bids (refer to Chapter 9 in [12]), as

$$
\left(c_{x}, v^{x}\right) O R\left(c_{y} A N D c_{z}, v^{y z}\right) O R \cdots
$$

Several separate parts are connected by OR operators. Each part is a bundle which in the form of either atomic bid or additive bid.

\section{Channel Allocation Mechanism in COMBINATORIAL AUCTION}

In this section we develop allocation mechanisms under different bidding formats to solve the problem defined in 2 .

\section{A. Single unit atomic bid}

The solution for this bid is straightforward. We simply sort the valuations on each channel in decreasing order and choose the highest bidder as the winner. In this way, the social welfare is maximized while the allocation constraints are followed.

\section{B. Multi-unit alternative bid}

In this bid the buyer requests one out of multiple channels. We can formulate the allocation problem under this format as Maximum Weighted Matching(MWM) problem in bipartite graph [13]. A bipartite graph is a graph whose vertices can be divided into two disjoint sets $U$ and $V$ such that every edge connects a vertex in $U$ to one in $V$, indicating the buyer set $\mathcal{S}$ and channel set $\mathcal{C}$ in our model. An edge exists between two vertices means the buyer put a request on the channel. The weight on the edge is a single valuation in buyer's bid. For instance, the buyer $j$ bids $\left(c_{1}, v_{j}^{1}\right) O R\left(c_{2}, v_{j}^{2}\right)$. Then in the graph, vertex $j$ have edges connecting to vertex $c_{1}$ and $c_{2}$ with weights $v_{j}^{1}$ and $v_{j}^{2}$, respectively.

The auctioneer forms the graph by mapping all the bundles in users' bids into edges. Then the auctioneer conducts the MWM algorithm to find Maximum weighted matching. Maximum weighted matching is defined as a matching where the sum of weights of the edges in the matching have a maximal value while no two edges share a common vertex. The property of matching achieves our goal while satisfies the constraints. Hence the winners are connecting vertices in the matching and the edges indicate the final allocation.

A simple example is shown in Fig. 2(b). There are 5 SUs and 3 channels, the SUs' bids is shown in Table 2(a). In mapping stage, we add edges and label the weights. The

\begin{tabular}{l|l}
$S U 1$ & $\left(c_{1}, 4\right)$ \\
\hline$S U 2$ & $\left(c_{1}, 5\right)$ OR $\left(c_{2}, 5\right)$ \\
\hline$S U 3$ & $\left(c_{1}, 4\right)$ \\
\hline$S U 4$ & $\left(c_{2}, 6\right)$ OR $\left(c_{3}, 4\right)$ \\
\hline$S U 5$ & $\left(c_{2}, 8\right)$ OR $\left(c_{3}, 7\right)$
\end{tabular}
(a) The SUs' bids

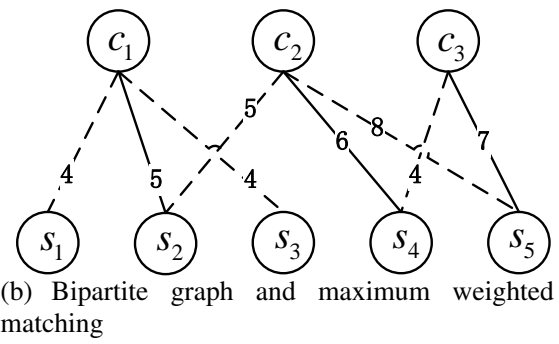

Figure 2: An illustration of example

graph is shown in Fig. 2(b), including both dash lines and solid lines. We apply MWM algorithm to find maximum weighted matching. The final matching is highlighted with solid line. The winning pairs include $\left(s_{2}, c_{1}\right),\left(s_{4}, c_{2}\right)$ and $\left(s_{5}, c_{3}\right)$, and social welfare is 18 . We can notice, though the edge between $c_{2}$ and $s_{5}$ owns the highest weight, it didn't appear in the final matching.

There exists many literatures on MWM, and it could be solved optimally with a time complexity $\mathrm{O}\left(\mathrm{V}^{2} \log \mathrm{V}+\mathrm{VE}\right)$, $\mathrm{V}$ and $\mathrm{E}$ is the number of vertices and edges in bipartite 
graph. The Hungarian algorithm is a popular polynomial-time algorithm to solve it optimally.

\section{Multi-unit additive bid}

In the literature, optimal auction methods to this problem normally have high computational complexity, which is prohibitive for real-time auction in dynamic spectrum access. We propose a computationally efficient near-optimal allocation mechanism based on a greedy algorithm.

The algorithm has three steps:

1) we transform the bid $b_{j}$ into virtual bid $v_{j} /\left|T_{j}\right| . v_{j}$ is the valuation of the bundle and $\left|T_{j}\right|$ is the number of channels in the bundle;

2) we sort all buyers' virtual bids in decreasing order and form a list $L$;

3) a greedy algorithm is used in the third step with the pseudo-code shown in Algorithm 1.

The algorithm examines every requests in $L$ sequentially, and grant the request if it does not overlap with previous granted requests. Otherwise, the valuation of current request is compared with sum of valuations of overlapped previous granted requests, and replace the overlapped requests if its valuation is higher. The process ends until all the request are examined.

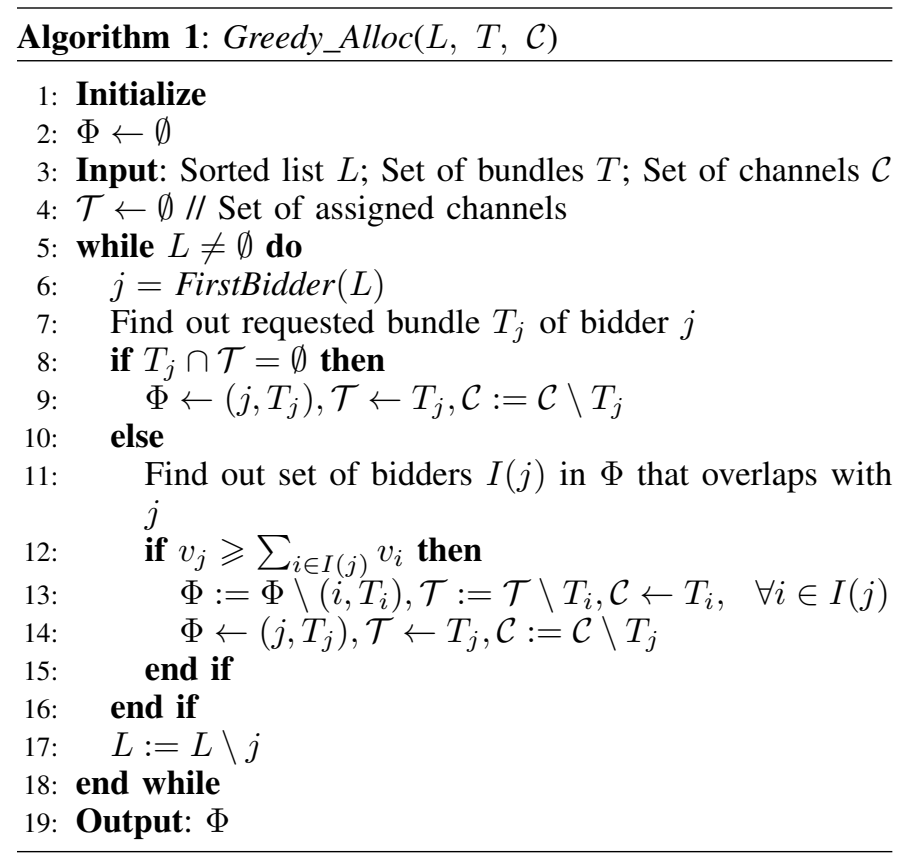

Next we analyze the approximation ratio of Algorithm 1:

Theorem 1. The proposed greedy algorithm can approximate the optimal allocation within a factor $\left(\frac{m}{n^{\prime}}-\epsilon\right)$, where $m$ is the number of channels and $n^{\prime}$ is the number of winning bidders that does not exist in the optimal solution. $\epsilon$ is a variable whose value is based on the ratio of common bidders in greedy solution and optimal solution.

Proof: Let $O P T$ be the set of winners in optimal allocation and $V^{*}=\sum_{i \in O P T} v_{i}$. Then we denote the result obtained by proposed algorithm as GRE and $V^{G}=\sum_{j \in G R E} v_{j}$. We now prove that

$$
V^{*} \leqslant\left(\frac{m}{n^{\prime}}-\epsilon\right) V^{G}
$$

Let us consider the winning bidders in $O P T$ and $G R E$, denote as $\left\{b_{1}^{*}, b_{2}^{*}, \ldots, b_{p}^{*}\right\}$ and $\left\{b_{1}^{G}, b_{2}^{G}, \ldots, b_{n^{\prime}}^{G}\right\}$, respectively. We can easily derive: there is no overlapping between bidders within $O P T$, i.e. $T_{i} \cap T_{i^{\prime}}=\emptyset \forall i, i^{\prime} \in O P T$, and so are the bidders in $G R E$; for every bidder in $G R E$, there must exist at least one bidder in $O P T$ overlap with it. If not so, we can put this bidder in $O P T$ and form a better solution, which is contrary to the assumption of $O P T$.

There may exist common bidders in both two sets. If so, without loss of generality, we remove these bidders and consider the worst case where $O P T$ and GRE have no bidders in common. Such operation does not affect the difference of valuations between $O P T$ and GRE. We inherit the denotation of $O P T$ and $G R E$ after removing common bidders.

We mix the bidders in $O P T$ and GRE, and rank them in decreasing order of $v_{i} /\left|T_{i}\right|$ as step 1 . In the greedy algorithm, we go through all those bidders. For any bidder $b_{i}^{*}, i \in O P T$, when it is considered in greedy algorithm, either it stays in $G R E$ temporarily and be replaced by a lower-rank bidder, or directly be rejected due to overlapping. In the former case, we consider the bidders in GRE. For example, assume the bidder $b_{j}^{G}$ overlaps with a number of bidders in $O P T$, denoted as $I(j)$. According to the proposed algorithm, there is

$$
\sum_{i \in I(j)} v_{i}^{*} \leqslant v_{j}^{G}
$$

In the later case, for any bidder $b_{i}^{*}$ in $O P T$, there would be a number of $b_{j}^{G}, j \in G R E$ overlapping with it, denoted as $I(i)$. According to the proposed algorithm, there is

$$
v_{i}^{*} \leqslant \sum_{j \in I(i)} v_{j}^{G}
$$

We divide all bidders in $O P T$ into the two cases and map them to (9) (10). Adding them together, we get a new inequality. The left part of the inequality equals $V^{*}$, the right part equals sum of $v_{j}^{G} \cdot k_{j}$, where $k_{j}$ is a constant. We should notice that at most $\left|T_{j}^{G}\right|$ will be associated with $b_{j}^{G}$, because no two bidders in $O P T$ intersect with each other. Then we have

$$
V^{*} \leqslant \sum_{j \in G R E} v_{j}^{G} \cdot\left|T_{j}^{G}\right| .
$$

According to Cauchy-Schwarz inequality, such that

$$
V^{*} \leqslant \sum_{j \in G R E} v_{j}^{G} \cdot\left|T_{j}^{G}\right| \leqslant \sqrt{\sum_{j \in G R E}\left(v_{j}^{G}\right)^{2}} \sqrt{\sum_{j \in G R E}\left(\left|T_{j}^{G}\right|\right)^{2}}
$$




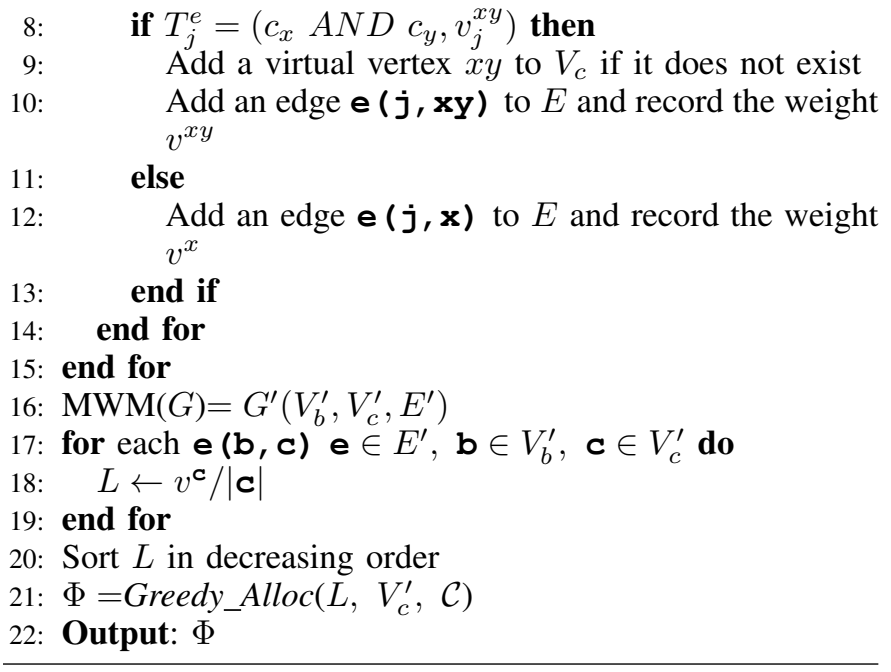

Equality holds if and only if $v_{j}^{G} /\left|T_{j}^{G}\right|=$ $v_{j^{\prime}}^{G} /\left|T_{j^{\prime}}^{G}\right|, \quad \forall j, j^{\prime} \in G R E$. Moveover, we represent the upper bound by setting $v_{j}^{G}=v_{j^{\prime}}^{G}=V^{G} / n^{\prime}\left|T_{j}^{G}\right|=\left|T_{j^{\prime}}^{G}\right|=$ $M^{G} / n^{\prime}, \forall j, j^{\prime} \in G R E$, and $M^{G}$ is the total number of allocated channels in greedy algorithm. Finally we have

$$
V^{*} \leqslant \sqrt{\left(\frac{V^{G}}{n^{\prime}}\right)^{2} \cdot n^{\prime}} \sqrt{\left(\frac{M^{G}}{n^{\prime}}\right)^{2} \cdot n^{\prime}}=\frac{M^{G}}{n^{\prime}} \cdot V^{G} \leqslant \frac{m}{n^{\prime}} \cdot V^{G}
$$

We should notice the approximation ratio $\frac{m}{n^{\prime}}$ only indicates the worst case. Taking removed common bidders into account, we can revise (14) as

$$
V^{*} \leqslant\left(\frac{m}{n^{\prime}}-\epsilon\right) \cdot V^{G},
$$

so that Theorem 1 is proved. The value $\epsilon$ is based on the ratio of common bidders in $O P T$ and GRE.

In the numerical evaluation, we compare our result with optimal value. It shows that the actual approximation ratio is close to the optimal one.

\section{Integrated bid}

An integrated bidding format can be reconstructed to a unified form, which enables the auctioneer to extract bundle information from the buyers' bids. We call reconstructed bids as trans-bids, denoted by $t r_{j}$. The bundles in trans-bid are connected by OR operator, in the form of either atomic bid or additive bid. To realize the constraint that each buyer gets at most one bundle in its request, we firstly map trans-bids into a bipartite graph and find out the optimal matching. Then we use the same method in Subsection IV-C to eliminate the overlapping on requests. The pseudo-code of the approximate allocation algorithm is shown in Algorithm 2.

Our algorithm consists of three steps:

1) we generate a bipartite graph based on trans-bids. The initial vertex sets in the graph are buyers and channels. We check each buyer's trans-bid sequentially. If the unit connected by OR is an atomic bid, we directly add an edge between buyer and the channel. If it is an additive bid, we add a virtual vertex in the channel vertex set and add an edge. The weight on the edge is the valuation of that bid. For instance, if the trans-bid of a buyer is $\left(c_{1}, v_{1}\right) O R\left(c_{2} A N D c_{3}, v_{2}\right)$, we add an edge between the buyer and channel $c_{1}$ with the weight $v_{1}$. Following we add a virtual node 23 in the channel vertex set, and add an edge between the virtual node and buyer. The weight on this edge is $v_{2}$. If a virtual node is already added, we could directly add an edge and record the weight;

2) we generate a bipartite graph. We apply the same method in Section IV-B to find maximum weighted matching. There may exist virtual nodes in optimal matching and there are overlapping on requests;

3) we use a greedy algorithm same as that in Section IV-C to eliminate the overlapping on requests. Note that although it may find an optimal matching in step 2 , we cannot guarantee the optimality of Algorithm 2 due to the limitation of the greedy algorithm.

\section{Numerical Evaluation}

In this section, we compare our algorithms with the optimal solution via numerical tests. The optimal value is obtained by Integer Programming module in LINGO ${ }^{1}$, in which Branchand-Bound algorithm is used. The performance metrics are social welfare and spectrum utilization ratio.

We need to emphasis that, under the alternative bid, the social welfare achieved by MWM method is optimal, and as long as the number of bidders is sufficient, the spectrum utilization ratio can always approximates 1 . Therefore we do not make a comparison for the alternative bid. The main focus is on the greedy algorithm for additive bid and the approximation algorithm for integrated bid. We set the number of channels for SUs to be $m=128$. The maximal requirement for any bidder is 10 bundles. The items in the bundle are also randomly selected among 1 to $m$. We further assume that the bidder's valuation for each bundle is uniformly distributed between [0,1] [7].

Under the additive bid, we assume the number of SUs varies from 100 to 500 at a step size of 50. Fig. 3 depicts the comparison between result of Algorithm 1 and optimal value on social welfare. We can see Algorithm 1 continually has a high approximation ratio, around $95 \%$ of the optimal value. Fig. 4 shows a similar trend on spectrum utilization ratio. we can notice, with the number of SUs increasing, the spectrum utilization ratio also increases gradually, but the marginal utility of utilization ratio from increasing 50 SUs is decreasing. We infer that, finally it would reach an upper bound and keep constant.

We set the number of SUs varies from 100 to 250 under integrated bid. In Fig. 5, we show the comparison on social welfare. The approximation ratio is lower than that of Algorithm 1, but always larger than 0.5. It means Algorithm 2 can achieve at least half of the optimal value on social welfare. Moreover Algorithm 2 also has a good performance on spectrum utilization ratio in Fig. 6.

\section{CONCLUSION AND FUTURE WORK}

In this paper, we first define four bidding formats to fully represent on SUs' requirements on the channel selection. We

\footnotetext{
${ }^{1}$ LINGO is an optimization modeling and solving software for linear, nonlinear and integer programming.
} 

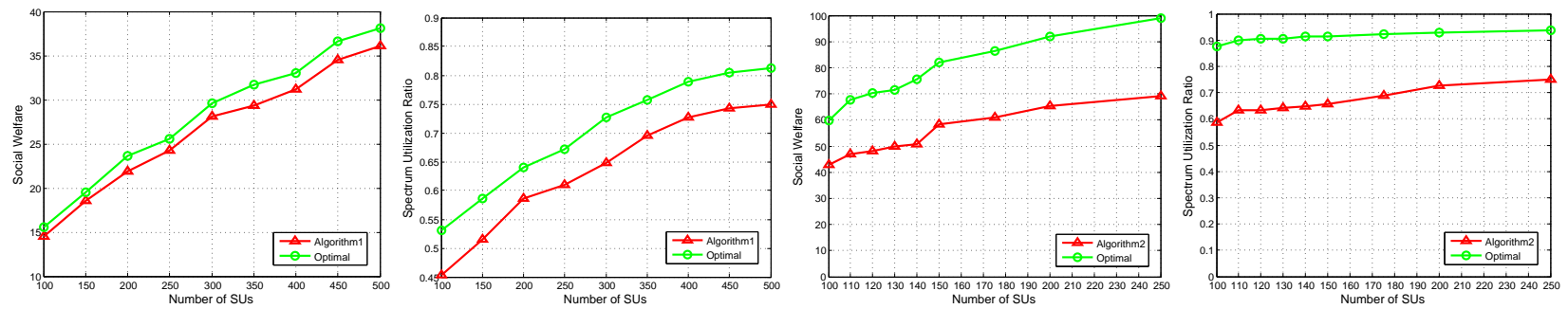

Figure 3: Comparison of so- Figure 4: Comparison of Figure 5: Comparison of so- Figure 6: Comparison of cial welfare under additive spectrum utilization ratio cial welfare under integrated spectrum utilization ratio bid under additive bid bid under integrated bid

propose algorithms to maximize social welfare for each biding format:under the alternative bid, we use MWM method to find the optimal match; under the additive bid, we design a greedy algorithm to maximize social welfare while satisfying the allocation constraints; under integrated bid, we introduce the process of reconstruction, then propose an approximation algorithm for the channel allocation. The approximation algorithm combines MWM and greedy algorithm, in which MWM is used for filtering bundles and the greedy algorithm used for eliminating overlapping. The numerical evaluation show that the proposed mechanism achieves at least $50 \%$ of the optimal value.

For the future work, we will refine the bidding language in combinatorial auction to CRN and develop auction algorithms with more flexibility on the channel selection and less computation complexity for the large bidding size.

\section{REFERENCES}

[1] Y. Zhang, D. Niyato, P. Wang, and E. Hossain, "Auction-based resource allocation in cognitive radio systems," Communications Magazine, IEEE, vol. 50, pp. $108-120,2012$.

[2] F. Wang, M. Krunz, and S. Cui, "Price-based spectrum management in cognitive radio networks," in CrownCom 2007. 2nd International Conference on, 2007, pp. $70-78$.

[3] T. Zhang and X. Yu, "Spectrum sharing in cognitive radio using game theory-a survey," in Wireless Communications Networking and Mobile Computing (WiCOM), 2010 6th International Conference on, 2010, pp. $1-5$.

[4] L. Chen, S. Iellamo, M. Coupechoux, and P. Godlewski, "An auction framework for spectrum allocation with interference constraint in $\operatorname{cog}$ nitive radio networks," in INFOCOM, 2010 Proceedings IEEE, 2010 , pp. $1-9$.

[5] H.-B. Chang and K.-C. Chen, "Auction-based spectrum management of cognitive radio networks," Vehicular Technology, IEEE Transactions on, vol. 59, pp. $1923-1935,2010$.

[6] H. Xu, J. Jin, and B. Li, "A secondary market for spectrum," in INFOCOM, 2010 Proceedings IEEE, 2010, pp. 1 -5.

[7] M. Dong, G. Sun, X. Wang, and Q. Zhang, "Combinatorial auction with time-frequency flexibility in cognitive radio networks," in INFOCOM, 2012 Proceedings IEEE, 2012, pp. $2282-2290$.

[8] B. Lehmann, D. Lehmann, and N. Nisan, "Combinatorial auctions with decreasing marginal utilities," in Proceedings of the $3 r d$ ACM conference on Electronic Commerce, 2001, pp. 18-28.

[9] D. Porter, S. Rassenti, A. Roopnarine, and V. Smith, "Combinatorial auction design," Proceedings of the National Academy of Sciences, pp. $11153-11157,2003$

[10] F. Gul and E. Stacchetti, "Walrasian equilibrium with gross substitutes," Journal of Economic Theory, pp. 95-124, 1999.

[11] S. de Vries and R. V. Vohra, "Combinatorial auctions: A survey," INFORMS J. on Computing, pp. 284-309, 2003.
[12] P.Cramton, Y.Shoham, and R.Steinberg, Combinatorial Auctions. MIT Press, 2006.

[13] D. B.West, Introdution to Graph Theory(2nd edition). Prentice Hall, 1999. 